\title{
Global Perspective of Novel Therapeutic Strategies for the Management of NeuroAIDS
}

\author{
Novel drug delivery methods for NeuroAIDS
}

https://doi.org/10.1515/bmc-2018-0005

received January 29, 2018; accepted April 6, 2018.

Abstract: Among Human immunodeficiency virus (HIV) infected individuals, around two-thirds of patients present with neuroAIDS, where HIV-associated neurocognitive disorders (HAND), and HIV-associated dementia (HAD) are the most prevailing neurological complications. The neuropathology of neuroAIDS can be characterized by the presence of HIV infected macrophages and microglia in the brain, with the formation of multinucleated giant cells. Global predominant subtypes of HIV-1 clade $B$ and $C$ infections influence the differential effect of immune and neuronal dysfunctions, leading to cladespecific clinical variation in neuroAIDS patient cohorts. Highly active antiretroviral therapy (HAART) enhances the survival rate among AIDS patients, but due to the inability to cross the Blood-Brain-Barrier (BBB), incidence of neuroAIDS during disease progression may be envisaged. The complex structure of blood-brainbarrier, and poor pharmacokinetic profile coupled with weak bio-distribution of antiretroviral drugs, are the principle barriers for the treatment of neuroAIDS. In the combined antiretroviral therapy (cART) era, the frequency of HAD has decreased; however the incidence of asymptomatic neurocognitive impairment (ANI) and minor neurocognitive disorder (MND) remains consistent. Therefore, several effective novel nanotechnology based

\footnotetext{
*Corresponding author: Shailendra K Saxena, Center for Advanced Research (CFAR)-Stem Cell/Cell Culture Unit, King George's Medical University (KGMU), Lucknow 226003, India; CSIR-Centre for Cellular and Molecular Biology, Uppal Road, Hyderabad 500007, India, E-mail: shailen@kgmcindia.edu

Swatantra Kumar, Vimal K Maurya, Himanshu R Dandu, Madan LB Bhatt: Center for Advanced Research (CFAR)-Stem Cell/Cell Culture Unit, King George's Medical University (KGMU), Lucknow 226003 , India
}

therapeutic approaches have been developed to improve the availability of antiretroviral drugs in the brain for the management of neuroAIDS.

Keywords: HIV; NeuroAIDS; HAD; HAND; HAART; BBB; AIDS; ART; Nanogels; Nanoemulsions; Liposomes; MNPs.

\section{Abbreviations}

HIV (Human Immunodeficiency Virus), Central Nervous System (CNS), Blood-Brain-Barrier (BBB), HIV Associated Neurocognitive Disorders (HAND), HIV Associated Dementia (HAD), Asymptomatic Neurocognitive Impairment (ANI), Minor Neurocognitive Disorder (MND), HIV Encephalitis (HIVE), AIDS Dementia Complex (ADC), Multinucleated Giant Cells (MGCs), Neuropsychological impairment (NPI), Trans-Activator of Transcription (Tat), Antiretroviral therapy (ART), cART (combined anti retroviral therapy), Anti-retroviral (ARV), Highly Active Antiretroviral Therapy (HAART), Macrophage-tropic HIV-1 R5 envelopes (mac-tropic HIV-1 R5 envs), P glycoprotein (P-gp), Nanoparticles (NPs), Nanotechnology Based Drug Delivery Systems (NDDS), Solid Lipid Nanoparticles (SLN), LipidDrug Conjugates(LDC), NanoStructuredLipidCarriers (NLC), Methylmethacrylate-sulfopropylmethacrylate (MMA-SPM), Poly Butyl Cyanoacryalate (PBCA), 3'-azido3'-deoxythymidine-5'-triphosphate (AZTTP), ProteinTransduction Domain (PTD), Magnetic Resonance Imaging (MRI), Magnetic nanoparticles (MNP), Brain Microvessel Endothelial Cells (BMVECs), Adsorptive Mediated Transcytosis (AMT), Mononuclear Phagocytes (MPs), Electromagnetic Interference (EMI), Frequency Modulated (FM) and Amplitude Modulated (AM)

\# Both the authors contributed equally.

Ә Open Access. (c) 2018 Swatantra Kumar et al., published by De Gruyter. (a) BY-NC-ND This work is licensed under the Creative Commons Attribution-NonCommercial-NoDerivatives 4.0 License. 


\section{Introduction}

Several novel therapeutic strategies have improved the care of people living with HIV/AIDS in the past three decades. HIV-1 infection of the central nervous system (CNS) leads to neuroAIDS in about $30-50 \%$ patients [1, 2], which may be characterized by a decline in brain function and movement skills, as well as shifts in behaviour and mood [3]. This disorder is called HIVassociated Neurocognitive Disorder (HAND), which is primarily diagnosed on the basis of brain functional status assessment and neurophysiological testing. HAND can be further categorized as asymptomatic HAND/ asymptomatic neurocognitive impairment (ANI), minor neurocognitive disorder (MND), and HIV associated dementia (HAD). In the combined antiretroviral therapy (cART) era, the frequency of HAD has decreased, however the incidence of ANI and MND remains consistent [4]. The prevalence of ANI and MND is $~ 50-80 \%$ (of the total HAND patients), whereas the prevalence of HAD is $2-4 \%$. [5]. ANI can be characterized by HIV associated cognitive impairment, and the involvement of at least two cognitive areas, such as memory, processing speed, attention, sensory perceptual and motor skills, which do not interfere with the daily activity of the patients. Similarly, MND can be characterized by HIV associated cognitive impairment, with a decline in brain function, movement skills, and shifts in behaviour. Whereas in HAD, no preexisting cause of dementia should be present, in addition to MND [6].

\section{Prevalence and Epidemiology of NeuroAIDS}

On the basis of differences in the genome, HIV-1 subtypes are phylogenetically classified in distinct groups $-\mathrm{M}, \mathrm{N}, \mathrm{O}$, and $\mathrm{P}$ and subtype/clade - A-K; and several recombinant forms ( 89). Subtypes A-D are highly prevalent, whereas other subtypes have low prevalence and limited geographic distributions. Two circulating recombinant forms (CRF01_AE and CRF02_AG) are found principally in Southeast Asia and West Africa [7]. The majority of clinical HIV research has been carried out for HIV-1 subtype B, predominantly in America, Western Europe and Australia, however, it only reflects $12 \%$ of global HIV infection. In contrast, less research has been conducted for the HIV-1 subtype C, which represents 50\% of global HIV infection. HIV-1 subtype C is predominant in Africa and India. The ART has been developed primarily to target HIV-1 subtype B [8]. However, ART has been shown to be ineffective against HIV-1 subtype C in Sweden [9]. Prevalence of HAD in the untreated HIV-1 subtypes A and D infected individuals is $~ 31 \%$ [10].

Clade-specific differences at the level of neurological manifestation can be explained by the difference in the amino acid sequence of viral regulatory protein Trans-activator of transcription (Tat) among the various subtypes of HIV-1 [11]. HIV-1 clade C infection in South African revealed that the cognitive impairment induced by clade $\mathrm{C}$ is similar to other HIV clades, and infection is independent of substitution of HIV Tat (C31S) [12]. HIV-1C in India and sub-Saharan Africa are underdiagnosed due to several reasons including; social stigma, shorter life expectancy, short survival of HIV infected individuals along with prevalence of opportunistic infections, all together decreasing the reported incidences of HAD. Global predominant subtypes of HIV-1 clade B and C infections influence the differential effect of immune and neuronal dysfunctions leading to clade-specific clinical variation in neuroAIDS patient cohorts [1].

\section{Neuropathology of NeuroAIDS}

A low or nadir CD4 count is the predictive factor for HAND and neuropsychological impairment (NPI) [13]. During HIV infection of the brain, gray and white matter gets impaired and can be observed in the brain autopsy of HIV infected samples. Moreover, the perivascular lymphocytic cuffing and low grade lymphocytic meningitis are identified, especially in the white matter comprising of primarily $\mathrm{CD}^{2} \mathrm{O}^{+} \mathrm{B}$ lymphocytes and $\mathrm{CD} 8^{+} \mathrm{T}$ lymphocytes [14]. Patients with HAD present an overall reduced volume of gray and white matter predominantly in the area of parietal, frontal and temporal lobes. Viral proteins, such as Tat, Vpr and Gp120, cause neuronal apoptosis by TNF- $\alpha$, IL-6 and IL-1, intracellular calcium ion load and increased ROS production [15]. Neuropathological alterations range from HIV encephalitis (HIVE), ADC (AIDS dementia complex), neurodegeneration, necrotizing lesions, neurosyphilis, meningitis, neuropathies, vacuolar myelopathy, leukoencephalopathy and CNS lymphomas [3]. The neuropathological features of HIVE have been demonstrated as the presence of multinucleated giant cells (MGCs) and white matter pallor, due to perivascular demyelination, as well as hippocampal neuronal apoptosis in companion with microglia activation and 


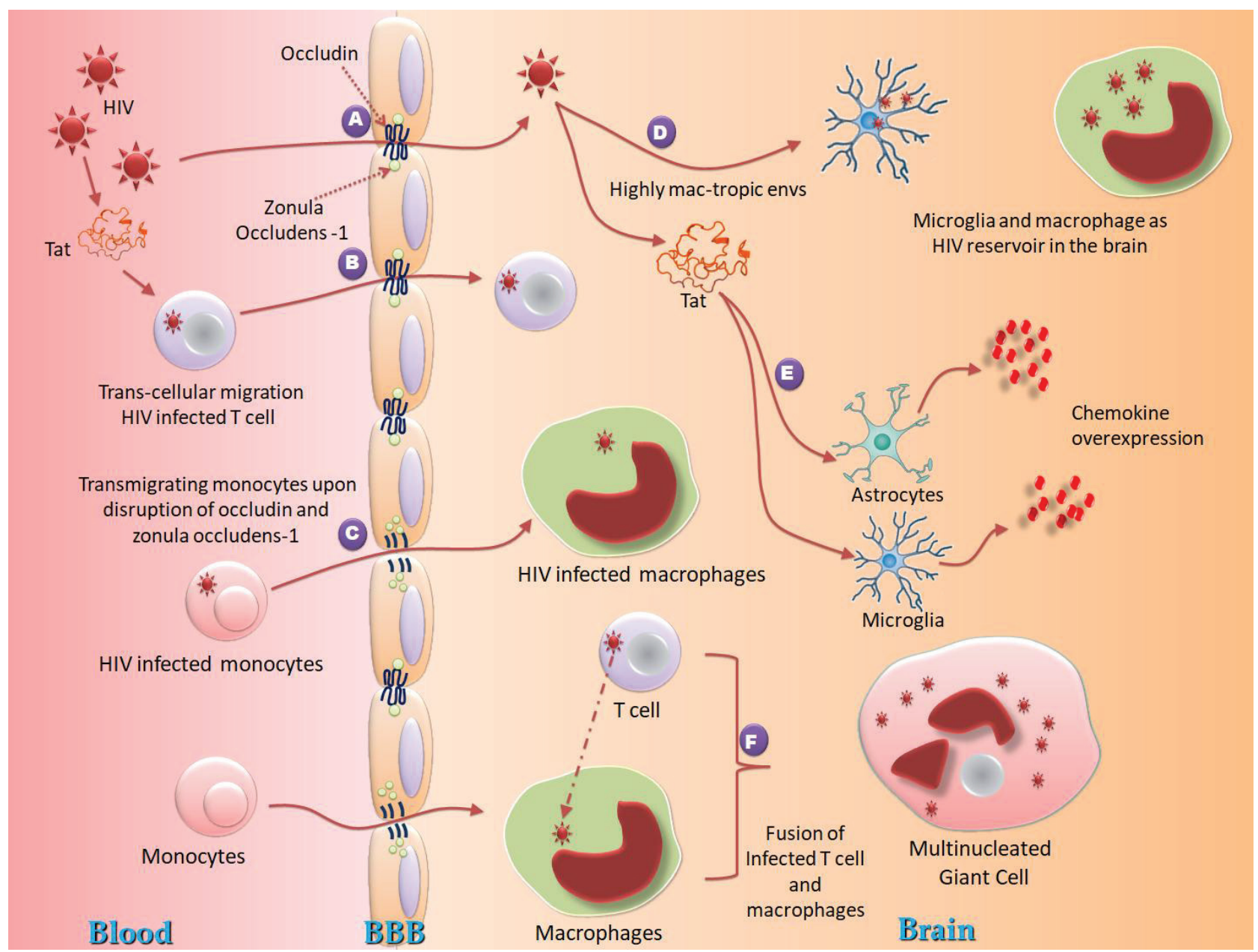

Figure 1: Mechanism of HIV Neuropathogenesis. (A) Cell independent transmigration of HIV across the BBB, (B) HIV-1 Tat protein functions as a growth factor resulting in the trans-cellular migration of HIV infected cells into the brain, (C) Disruption of endothelial tight junction proteins, specifically occludin and zonula occludens-1, results in transmigration of monocytes/macrophages into the brain, (D) The presence of highly mac-tropic HIV-1 R5 envs in the brain tissue is responsible for the adaption for replication in microglia and macrophages, which in turn forms the principal HIV reservoir. (E) Viral Tat protein induces the over-expression of chemokines by astrocytes and microglia, which are responsible for the leukocyte entry to CNS, (F) Interaction between infected T cells and macrophages leading to cell fusion and the generation of highly virus productive multinucleated giant cells (MGCs).

astrocytosis. Increased proliferation of progenitor cells in the subgranular zone and hippocampal dentate granule cell layer have been observed in HIVE brain autopsy sample [14].In HIVE patients, Tat mRNA and protein has been detected in the brain tissue extract [16]. Tat has been shown to cross the BBB via a nonsaturable mechanism with a unidirectional influx rate of about $0.490 \mathrm{microl} / \mathrm{gram} /$ min, where $0.126 \%$ of an intravenous dose of Tat enters each gram of the brain. The most Tat-permeable regions of the brain are the hypothalamus, occipital cortex, and hippocampus [17]. Tat has been detected in $42 \%$ of CSF samples by ELISA from HIV-infected individuals [18].

\section{Mechanism of Neuropathogenesis and NeuroAIDS}

Transmigration of HIV across the BBB (Figure-1) can be explained by several mechanisms [19]. Disruption of endothelial tight junction proteins, specifically occludin and zonula occludens-1, leads to monocyte/macrophage infiltration into the brain [20]. HIV Tat protein induces the over-expression of chemokines by astrocytes and microglia, which are responsible for the leukocyte entry to CNS [21]. Similarly, Gp120 is critically involved in the neuropathogenesis of HIV by inducing injury and apoptosis [22]. An astonishing role of Tat protein as a 
growth factor has been proposed, which results in the trans-cellular migration of HIV infected cells into the CNS [23]. Surprisingly, neuroAIDS is independent of HIV titer and is correlated with the degree of monocyte infiltration, following activation of microglia in the brain [24]. CD4 expressing cells with either CXCR4 or CXCR5 are targeted by HIV-1. HIV-1 isolates targeting CXCR5 predominantly infects macrophages, hence classified as M-tropic; whereas isolates targeting CXCR4 predominantly infects T cells, classified as T-tropic [25]. Macrophage-tropic (mactropic) HIV-1 R5 strains are known to infect macrophages with low CD4 expression, whereas non-macrophagetropic (non-mac-tropic) HIV-1 R5 variants requires high level of CD4 expression and primarily target $\mathrm{CD} 4^{+} \mathrm{T}$ Cells [26]. Highly macrophage-tropic HIV- 1 R5 variants are predominant in brain tissue and can be detected in CSF, whereas the detection in immune tissue or the blood is infrequent, even in the late disease condition [27]. The presence of highly macrophage-tropic HIV-1 R5 envelopes (mac-tropic HIV-1 R5 envs) in brain tissue is responsible for adaption for replication in microglia and macrophages, which in turn forms the principal HIV reservoir. Moreover, the mechanism behind the virus dissemination from HIV-1 infected macrophages, and establishment of virus reservoir, can be explained as cell to cell contacts between infected $\mathrm{T}$ cells and macrophages, leading to cell fusion for rapid and massive transfer of R5 tropic viruses to macrophages. These fused cells attain the ability to further fuse with neighbouring non infected macrophages, eventually leading to the generation of MGCs [28].

\section{Challenges for the treatment}

More than 35 million people infected with HIV are living worldwide. In 2013, access to ART treatment reached 12.9 million people ( $37 \%$ of 35 million), which further enhanced to 15 million people by 2015 . The UNAIDS proposed 90-90-90 target by 2020, where $90 \%$ of the HIV infected individual will know their status, $90 \%$ of HIV diagnosed people will have access to ART and $90 \%$ of those on ART will be virally suppressed [29]. ART has been significantly executed among the HIV infected individuals [30], even though the eradication of HIV from the CNS remains a challenging task. Highly active antiretroviral therapy (HAART) enhances the survival rate among the AIDS patients, but due to inability to cross the BBB, incidence of neuroAIDS during the disease progression may be envisaged [31]. Upon administration, ART mostly bound with plasma proteins, further limiting their available fractions for the CNS [32]. The complex structure of the BBB and poor pharmacokinetic profile, coupled with weak bio-distribution of antiretroviral drugs, are the principal barriers for the effective drug delivery to the CNS [33]. Brain microvessel endothelial cells (BMVECs), contributing to the formation of brain capillaries, exert a major obstacle for molecule passage. BMVECs acquire a higher amount of mitochondria and low pinocytotic activity, leading to diminished transport of anti-retroviral (ARV) drugs across the BBB. In addition, overexpression of P glycoprotein (P-gp) on the BBB limits the entry of many drugs, such as protease inhibitors (indinavir and ritonavir), epileptic drugs, and anti-inflammatory agents [34]. The mechanism of neuroAIDS has been well explored, despite the treatment challenges that remain as a major obstacle for the development of effective therapeutics [35]. Consequently, several novel therapeutic approaches have been developed to improve the antiretroviral drugs delivery to the brain for the management of neuroAIDS and various other CNS disorders with desired properties [36].

\section{Novel drug delivery approaches for targeting brain}

The ineffectiveness of neurotherapeutics for CNS infections and disorders can be explained by the unsuccessful delivery of the drugs across the BBB. Though there is the presence of a comparative high blood flow in the brain, the drug delivery to the brain remains a challenging task [37]. Therefore, advancement of approaches exploiting novel therapeutics which specifically target the brain, with enhanced drug penetration and effectiveness, is imperative. Several nanotechnology-based approaches have been utilized for effective drug delivery to the CNS (Figure-2), where the most significant approaches are polymeric nanocarriers, liposome-based nanomedicines, magnetic nanoparticles-based nanomedicines, and nanogels [38]. In addition to nanotechnology, alteration of the BBB, adsorptive mediated transcytosis by BMVECs and cell-mediated delivery, have been shown to be effective methods for ARV delivery across the BBB [39]. Similarly, other approaches include chemical modification of proteins for CNS delivery, prodrug-based approach, and biotechnology-based therapeutics for improved permeability and therapeutics delivery to the brain [40]. 


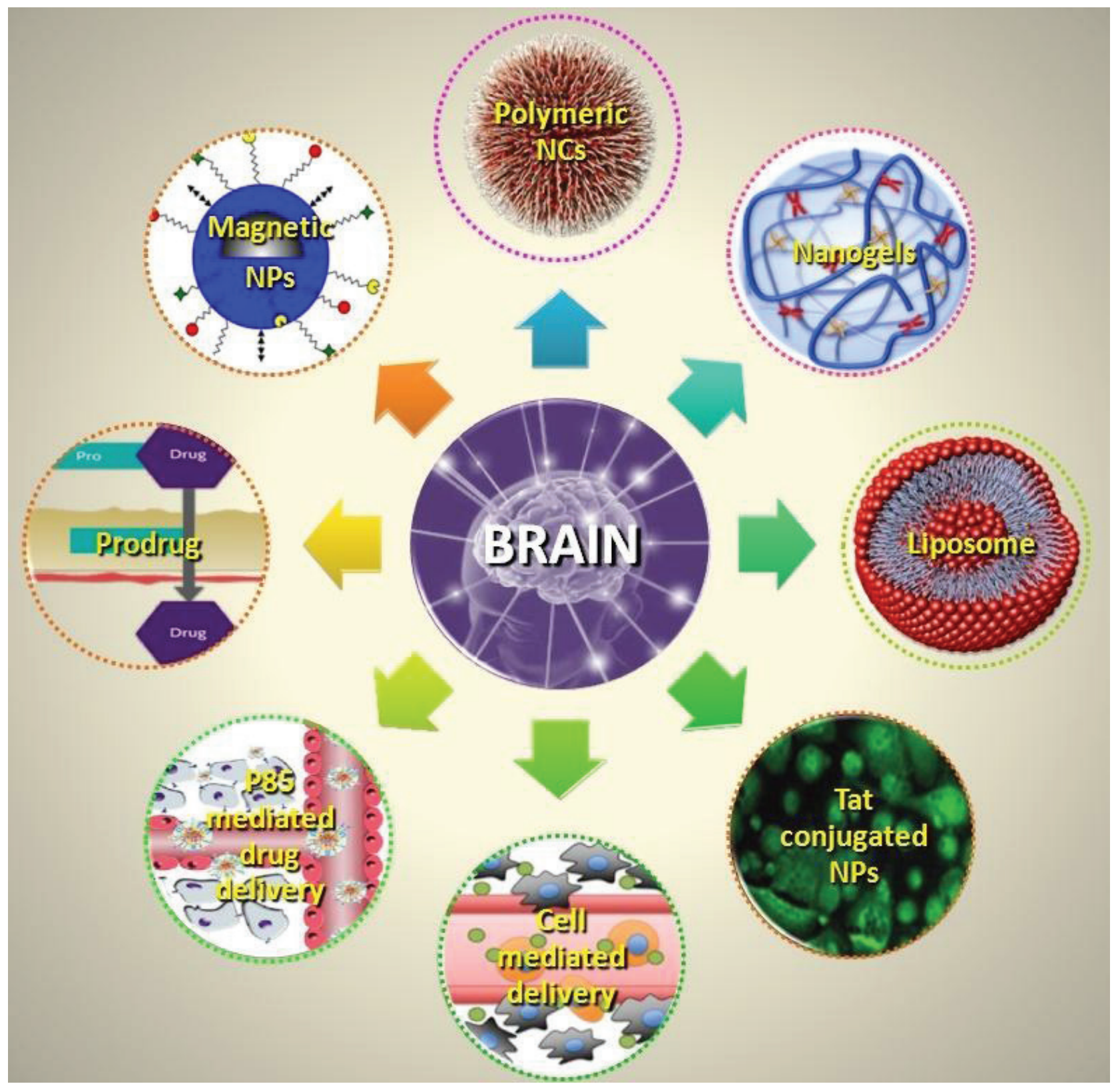

Figure 2: Novel drug delivery approaches for targeting the brain in NeuroAIDS. Novel drug delivery approaches for targeting the brain in NeuroAIDS are Magnetic nanoparticles-based Nanomedicines (3'-azido-3'-deoxythymidine-5'-triphosphate), Polymeric Nanocarriers (lamivudine, stavudine and zidovudine), Nanogels (zidovudine and didanosine), Liposome-based Nanomedicines (zidovudine, zalcitabine and didanosine), Tat conjugated NPs mediated drug delivery (ritonavir), Cell mediated delivery of NPs (ritonavir and efavirenz), P85 mediated drug delivery (lamivudine, zidovudine and nelfinavir) and Prodrug-based approach (tenofovir disoproxil fumarate and fosamprenavir).

\section{Nanotechnology-based approach}

Advancement of current ARV drugs using nanotechnology can be achieved with higher effectiveness and reduced side effects, ultimately achieving therapeutic concentration at the target sites. Properties, such as multi-functionality, small particle size, increased surface area, high dissolution rate, have increased the permeability of nanocarriersacross the BBB. Nanotechnology-based drug delivery systems (NDDS) offer various strategies, which can be exploited for the development of various nanoformulations based therapy [41]. These nanoformulations include solid lipid nanoparticles (SLN), polymeric nanoparticles, nanogels, nanoemulsions, nanosuspensions, nanospheres, 
Table 1: Nanotechnology based CNS delivery of antiretroviral drugs.

\begin{tabular}{|c|c|c|c|c|}
\hline Class & Category & Mechanism of Action & Drugs & Nanomaterials/nanocarriers \\
\hline I & $\begin{array}{l}\text { Entry and Fusion } \\
\text { inhibitors }\end{array}$ & $\begin{array}{l}\text { Drugs interfere with the, gp41-mediated fusion process. } \\
\text { They block the CCR5 coreceptor that works together with } \\
\text { gp } 41 \text { to facilitate HIV entry through the membrane into } \\
\text { the cell.HIV may express either the CCR5 coreceptor, or } \\
\text { the CXCR4 coreceptor, or both. }\end{array}$ & $\begin{array}{l}\text { Enfuvirtide, } \\
\text { Vicriviroc, } \\
\text { Maraviroc, } \\
\text { Aplaviroc }\end{array}$ & Magnetic nanoparticle \\
\hline II & Protease inhibitors & Reversible inhibition of HIV aspartyl protease & $\begin{array}{l}\text { Ritonavir, } \\
\text { Indinavir, } \\
\text { Saquinavir, } \\
\text { Tipranavir }\end{array}$ & PLGA nanoparticles, Liposome \\
\hline III & Integrase inhibitors & $\begin{array}{l}\text { Specific inhibition of the final step in integration of stand } \\
\text { transfer of the viral DNA into host cell DNA. }\end{array}$ & $\begin{array}{l}\text { Raltegravir, } \\
\text { Elvitegravir, } \\
\text { Dolutegravir }\end{array}$ & Polymeric nanoparticles \\
\hline IV & $\begin{array}{l}\text { Nucleoside reverse } \\
\text { transcriptase } \\
\text { inhibitors (NRTI) }\end{array}$ & $\begin{array}{l}\text { NRTI inhibits the viral reverse transcriptase enzyme } \\
\text { which is responsible for transcribing viral RNA into } \\
\text { double stranded DNA. }\end{array}$ & $\begin{array}{l}\text { Zidovudine, } \\
\text { Zalcitabine, } \\
\text { Stavudine, } \\
\text { Lamivudine, } \\
\text { Abacavir }\end{array}$ & PLGA nanoparticles, Liposome \\
\hline $\mathbf{v}$ & $\begin{array}{l}\text { Non-nucleoside } \\
\text { reverse transcriptase } \\
\text { inhibitors (NNRTI) }\end{array}$ & $\begin{array}{l}\text { Directly inhibits HIV reverse transcriptase so that } \\
\text { selective inhibition of the replication of viral DNA occurs. }\end{array}$ & $\begin{array}{l}\text { Delavirdine, } \\
\text { Efavirenz, } \\
\text { Nevirapine, } \\
\text { Rilpivirine }\end{array}$ & $\begin{array}{l}\text { Tat-conjugated nanoparticle, } \\
\text { Solid lipid nanoparticles (SLN) }\end{array}$ \\
\hline
\end{tabular}

nanomicelles and liposomes, lipid drug conjugates (LDC) and nanostructured lipid carriers (NLC). Nanosizing of ARV drugs in the form of nanoparticles, nanocrystals or nanosuspensions, also increases the bioavailability and dissolution rate across the BBB (Table-1) [42].

\section{Polymeric nanocarriers for anti-retroviral drug delivery}

Polymeric Nanocarriers (Polymeric NCs) have been widely investigated for brain targeting. High encapsulation efficiency, stability, biodegradability, safety and physical properties such as permeability, the rate of disassociation, and simple modulation of release kinetics, all together promote the drug entry to the brain [43]. Polymers, such as methylmethacrylate-sulfopropylmethacrylate (MMA-SPM) and poly butyl cyanoacrylate (PBCA), have been extensively used for lamivudine, stavudine and zidovudine delivery for in vitro and in vivo models of neuroAIDS [44].

\section{Liposome-based nanomedicines}

Lipid-based nanocarriers have significant importance in drug delivery methods. Several advantages associated with lipid-based nanomedicines are cell specificity, low immunogenicity, and increased stability for targeting various sites of the body, including the brain, that makes them more effective and safe [45]. Being lipophilic in nature, liposomes can be considered as a vital strategy for ARV drugs, which have limited permeability to the CNS. Liposomes primarily developed for the delivery of hydrophobic anti-HIV drugs, such as zidovudine, zalcitabine, and didanosine, is not possible with other nanocarriers like solid lipid or magnetic nanoparticles [46].

\section{Magnetic nanoparticles-based nanomedicines}

Magnetic nanoparticles (MNPs) have been well explored for targeted drug delivery rationale. However, MNPs need to be considered as an effective drug delivery to the brain, as it possesses distinctive properties over others. Magnetic materials, such as Magnetite $\left(\mathrm{Fe}_{4} \mathrm{O}_{4}\right)$ and maghemite $\left(y-F e 2 \mathrm{O}_{3}\right)$, are the frequently used MNPs in the field of medicine [47]. The superparamagnetism property of MNPs can be extensively utilized in various approaches, such as magnetic resonance imaging (MRI) and magnetometry, whilst also making MNPs superior over other drug delivery methods, like polymeric nanoparticles, micelles, and liposomes. MNPs tagged with 3'-azido-3'- 
deoxythymidine-5'-triphosphate (AZTTP) have been shown to effectively cross the BBB without affecting the integrity of the BBB [48].

\section{Nanogels for anti-retroviral drug delivery}

Nanogels appear to be an excellent drug delivery system for modern therapeutics [49]. Conventional therapeutics have poor stability in the biological system, and nanogels enables selective uptake into targeted cells. Nanogels are designed for both hydrophilic and hydrophobic drug delivery, with polymeric matrices and particles having high absorption capacity. Nanogels have vital properties, including excellent biocompatibility and degradability profile, colloidal stability, swelling property in aqueous media, higher drug loading capacity, particle size (20-200 $\mathrm{nm})$, electromobility, and a non-immunologic response, that makes them a robust strategy for drug delivery in NeuroAIDS. Amphiphilic cationic nanogels have been used as a carrier for brain delivery for nucleoside reverse transcriptase inhibitors, such as zidovudine (AZT) and didanosine (ddI) [50].

\section{Cell-mediated delivery of nanoparticles across the BBB}

Dendritic cells, monocytes, and macrophages are mononuclear phagocytes (MPs), which are suitable for cell-mediated delivery via incorporating nanosized ARV drugs [51]. MPs can easily cross the BBB via diapedesis and chemotaxis movement, and reach the inflamed area in the brain. A range of nanoparticles (NPs) such as gelatin NPs, dendrimers, lipid-drug liposomes polymer blend NPs, and nanocrystals have been designed for the cellular uptake by MPs. Nanosuspensions containing ritonavir and efavirenz has been used for cell-mediated delivery across the BBB.

\section{Tat conjugated nanoparticles mediated drug delivery across the BBB}

TwodistincthalvesareexertedbyBMVECs, abluminal(brain) and luminal (blood). Adsorptive mediated transcytosis (AMT) by BMVECs provides an inimitable opportunity for ARV drug delivery across the BBB [52]. Bypassing the P-gp efflux action, Tat conjugated NPs have been shown to enhance the transport of encapsulated ritonavir via transcytosis [53]. Astonishing levels of encapsulated ritonavir in the CNS can be attained for a sustained period by using conjugated Tat-NPs compared to the free drugs. The drug clearance also had unsatisfactory limits. However, due to several limitations, such as immunogenicity and neurotoxicity, possessed by Tat, this strategy is poorly translated from research to patients. Adsorptive mediated transcytosis can be translationally advantageous if the post-release mechanisms and interactions between Tat-NPs and neurons are further explored.

A protein-transduction domain (PTD) is imperative for cellular delivery of nanoparticles, polypeptides, and polynucleotide, and are derived from Tat, DNAbinding protein VP22 (HSV-1) or some other proteins [54]. Introduction of PTD in a protein via chemical conjugation or by various other strategies can be extensively used for modification of many proteins and enables them to cross the BBB [55]. Point modification of proteins with small fatty acids, such as palmitate or stearate, is an important approach that increases the lipophilic nature and facilitates the CNS entry and cellular uptake of proteins [56].

\section{P85 mediated drug delivery across the BBB}

Alteration of the BBB permeability can be considered as an alternative approach, where molecules can be allowed to pass via the BBB. Nevertheless, this might be an unsafe practice because of the unwanted passage of blood components toward the CNS. Various strategies have been exploited for altering the permeability of the BBB, such as, osmotic, chemical, biochemical, and MRI guided ultrasound [57]. Electromagnetic interference (EMI) in the form of frequency modulated (FM) and amplitude modulated (AM) waves can be applied to effectively parcel the ARV containing nanoparticles across the BBB. Using EMI, SLNs and Polymeric NPs containing saquinavir have been shown to cross the monolayer of human BMVECs mimicking the BBB [58]. Moreover, square AM waves show the strongest effect by increasing the BBB permeability of NPs by more than 14 times, whereas, the permeability of free drugs are unaffected. Likewise, modulation of P-gp via energy depletion methods might effectively increase the ARV drug delivery across the BBB. Pluronic ${ }^{\circledR}$ P85 (P85), is a copolymer block of amphiphilic nature, comprising of two hydrophilic polyethylene oxide chains flanking a hydrophobic group of polypropylene oxide. P85 has been shown to increase the lamivudine, zidovudine and nelfinavir delivery across the $\mathrm{BBB}$, via inhibiting P-gp efflux activity [59]. Surprisingly, P85 also exhibits an antiretroviral effect in conjunction with P-gp modulating agent. 


\section{Prodrug-based approaches for drug delivery across the BBB}

With the advantage of better penetration, site specificity, and lower toxicities than the original drug, prodrugs actin an enzyme-dependent manner [60]. The BBB possesses several enzymes, which induce biotransformation of prodrugs into active an drug, eventually leading to transmigration of the drug across the BBB, with effective concentration at the site of action [61]. Tenofovir disoproxil fumarate and fosamprenavir are two prodrugs developed with similar antiviral efficacy and better penetration for the treatment of neuroAIDS [62].

\section{Conclusions}

NeuroAIDS remains a major challenge among AIDS individuals. Treatment schedules for patients are unsuccessful, due to the inability of continuous lifelong medication, which results in a low concentration of the drug and increases the possibility of high viral load in the CNS. Insufficient passage of ARV drugs across the BBB eventually reduces the effectiveness and persistence of resistant viral strain against HAART treatment, suggesting that we need to look forward to novel drug delivery strategies. The development of nanotechnologybased strategies allowed us to combat the complex neuropathology associated with neuroAIDS. Advancement of nanoformulations for encapsulation of ARV drugs has been designed for the transport of drugs across the BBB. Polymeric nanocarriers, nanogels, liposome-based nanomedicines, prodrug-based approach, chemical modification of proteins for CNS delivery, and magnetic nanoparticles-based nanomedicines are the various approaches which can be utilized for designing and targeting the CNS of neuroAIDS patients. Persistence of the macrophage-tropic $\mathrm{R} 5$ variants in the brain illustrates the associated challenges for the effective treatment, in order to completely eradicate the HIV. Recent studies are suggesting that several approaches, such as antiretroviral therapy intensification shock and kill approach, stem cell transplantation, and gene therapy can be used for the eradication of HIV. Other approaches, such as the use of JAK inhibitors (Ruxolitinib), ribonucleoside chain terminators, and NADPH oxidase inhibitors are also beneficial in the elimination of HIV from the CNS. Additionally, various biotechnology-based approaches include, stem cell therapy, CRISPR/Cas9 pathways, RNA interference, intrabodies and monoclonal antibodies. Novel drug delivery systems should be utilized for the effective drug delivery in neuroAIDS patients.

\section{Future perspectives}

The CNS infection and involvement during HIV/AIDS is still a complex obscurity, and needs to be explored extensively. In order to understand HIV associated neuropathogenesis and to determine the potentially promising drug targets, we need to develop various in vitro models to study the neuroinvasion of HIV. Global predominant subtypes of HIV-1 clade B and C infections influence the differential effect of immune and neuronal dysfunctions, leading to clade-specific clinical variation in neuroAIDS patient cohorts. However, clinical impairment rates in India, Thailand, and the USA, were found to be similar, suggesting further studies are required to ascertain the clade-specific differential effect of immune and neuronal dysfunctions leading to neuroAIDS. In addition, neurocognitive evaluation needs to be taken into consideration of local, socioeconomic, genetic and epidemiological factors, to determine the rates of HAD/HAND. The HAART regimens being utilized by AIDS patients needs to be considered, while assessing the impact that HIV-1 clades associated neurocognitive impairment. One of the principal reasons for the failure of neuroAIDS treatment is the inability of ART drugs to cross the BBB, leading to poor bioavailability in the CNS. The presence of highly mac-tropic HIV-1 R5 envs in the brain tissue is responsible for adaption for replication in microglia and macrophages, which in turn forms the principal HIV reservoir. Furthermore, mactropic HIV-1 R5 envs is responsible for the formation of compartmentalized clusters in the brain tissue of the patients with non-neurological complications. Persistence of the macrophage-tropic R5 variants in the brain illustrates the associated challenges for the effective treatment in order to completely eradicate HIV. Utilizing novel drug delivery methods involving antiretroviral drugs can be further enhanced for the effective treatment of neuroAIDS. These novel methods should undergo $e x$ vivo and in vivo studies to ascertain the neurotoxicity and effectiveness in neuroAIDS patients. Robust data to support these technologies is required to affirm their use in humans; hence there is a need to execute inter and intracontinental clinical trials as per the standards of variousinternational agencies. Quantitativeassessmentfor the neurocognitive impairment should be standardized in HIV infected individuals for understanding the prevalence of neuroAIDS. A development of prognostic markers based on signature sequences of clade-specific HIV is a priority area of research, which would help the clinicians for the early diagnosis of neuroAIDS. HAD/HAND are the most prevalent neurological complications of neuroAIDS, which is coupled with major challenges, and we need to proceed with a sense of urgency in this context. 
Acknowledgements: The authors are grateful to the Vice Chancellor, King George's Medical University (KGMU), Lucknow and Director, Centre for Cellular and Molecular Biology, Council of Scientific and Industrial Research (CSIR-CCMB), India for the encouragement and support for this work. SK Saxena is also supported by CCRH, Government of India, and US NIH grants: R37DA025576 and R01MH085259. The authors have no other relevant affiliations or financial involvement with any organization or entity with a financial interest in or financial conflict with the subject matter or materials discussed in the manuscript apart from those disclosed.

Conflict of interest statement: The authors declare no conflicts of interest.

\section{References}

1. Gelman BB, Endsley J, Kolson D. When do models of NeuroAIDS faithfully imitate "the real thing"? J Neurovirol. 2017;18:1-10.

2. Saxena SK, Tiwari S, Nair MP. A global perspective on HIV/ AIDS. Science. 2012;337(6096):798.

3. Sagar V, Pilakka-Kanthikeel S, Martinez PC, Atluri VSR, Nair $M$. Common gene-network signature of different neurological disorders and their potential implications to neuroAIDS. PLoS One. 2017;12(8):e0181642.

4. Saylor D, Dickens AM, Sacktor N, Haughey N, Slusher B, Pletnikov M, Mankowski JL, Brown A, Volsky DJ, McArthur JC. HIV-associated neurocognitive disorder-pathogenesis and prospects for treatment. Nat Rev Neurol. 2016;12(4):234-248.

5. Gott C, Gates T, Dermody N, Brew BJ, Cysique LA. Cognitive change trajectories in virally suppressed HIV-infected individuals indicate high prevalence of disease activity. PLoS One. 2017;12(3):e0171887.

6. Sanmarti M, Ibáñez L, Huertas S, Badenes D, Dalmau D, Slevin M, Krupinski J, Popa-Wagner A, Jaen A. HIV-associated neurocognitive disorders. J Mol Psychiatry. 2014;2(1):2.

7. Hemelaar J. The origin and diversity of the HIV-1 pandemic. Trends Mol Med. 2012;18(3):182-192.

8. Geretti AM, Harrison L, Green H, Sabin C, Hill T, Fearnhill E, Pillay D, Dunn D, UK Collaborative Group on HIV Drug Resistance. Effect of HIV-1 subtype on virologic and immunologic response to starting highly active antiretroviral therapy. Clin Infect Dis. 2009;48(9):1296-1305.

9. Häggblom A, Svedhem V, Singh K, Sönnerborg A, Neogi U. Virological failure in patients with HIV-1 subtype $C$ receiving antiretroviral therapy: an analysis of a prospective national cohort in Sweden. Lancet HIV. 2016;3(4):e166-e174.

10. Sacktor N, Robertson K. Evolving clinical phenotypes in HIVassociated neurocognitive disorders. Curr Opin HIV AIDS. 2014;9(6):517-520.

11. Mishra M, Vetrivel S, Siddappa NB, Ranga U, Seth P. Clade-specific differences in neurotoxicity of human immunodeficiency virus-1 B and C Tat of human neurons: significance of dicysteine C30C31 motif. Ann Neurol. 2008;63(3):366-376.
12. Buch S, Chivero ET, Hoare J, Jumare J, Nakasujja N, Mudenda V, Paul R, Kanmogne GD, Sacktor N, Wood C, Royal W, Joseph J. Proceedings from the NIMH symposium on "NeuroAIDS in Africa: neurological and neuropsychiatric complications of HIV". J Neurovirol. 2016;22(5):699-702.

13. Ellis RJ, Badiee J, Vaida F, Letendre S, Heaton RK, Clifford D, Collier AC, Gelman B, McArthur J, Morgello S, McCutchan JA, Grant I. CD4 nadir is a predictor of HIV neurocognitive impairment in the era of combination antiretroviral therapy. AIDS. 2011;25(14):1747-1751.

14. Tauber SC, Staszewski O, Prinz M, Weis J, Nolte K, Bunkowski S, Brück W, Nau R. HIV encephalopathy: glial activation and hippocampal neuronal apoptosis, but limited neural repair. HIV Med. 2016;17(2):143-151.

15. Xu Y, Kulkosky J, Acheampong E, Nunnari G, Sullivan J, Pomerantz RJ.HIV-1-mediated apoptosis of neuronal cells: Proximal molecular mechanisms of HIV-1-induced encephalopathy. Proc Natl Acad Sci U S A. 2004;101(18):70707075.

16. Hudson L, Liu J, Nath A, Jones M, Raghavan R, Narayan O, Male D, Everall I. Detection of the human immunodeficiency virus regulatory protein tat in CNS tissues. J Neurovirol. 2000;6(2):145-155.

17. Banks WA, Robinson SM, Nath A. Permeability of the bloodbrain barrier to HIV-1 Tat. Exp Neurol. 2005;193(1):218-227.

18. Johnson T, von Geldern G, Tyagi R, Sacktor N, McArthur J, Letendre S, Hasbun R, Petito C, Roh D, Verma A, Nath A. Detection of HIV-Tat protein in brain and CSF of patients on antiretroviral therapy. J Neurovirol 2013;19:S40-S41.

19. Zayyad Z, Spudich S. Neuropathogenesis of HIV: from initial neuroinvasion to HIV-associated neurocognitive disorder (HAND). Curr HIV/AIDS Rep. 2015;12(1):16-24.

20. Nakamuta S, Endo H, Higashi Y, Kousaka A, Yamada H, Yano M, Kido H. Human immunodeficiency virus type 1 gp120mediated disruption of tight junction proteins by induction of proteasome-mediated degradation of zonula occludens- 1 and -2 in human brain microvascular endothelial cells. J Neurovirol. 2008;14(3):186-195.

21. Eugenin EA, Dyer G, Calderon TM, Berman JW. HIV-1 tat protein induces a migratory phenotype in human fetal microglia by a CCL2 (MCP-1)-dependent mechanism: possible role in NeuroAIDS. Glia. 2005;49(4):501-510.

22. Bardi G, Sengupta R, Khan MZ, Patel JP, Meucci O. Human immunodeficiency virus gp120-induced apoptosis of human neuroblastoma cells in the absence of CXCR4 internalization. J Neurovirol. 2006;12(3):211-218.

23. Mishra M, Taneja M, Malik S, Khalique H, Seth P. Human immunodeficiency virus type 1 Tat modulates proliferation and differentiation of human neural precursor cells: implication in NeuroAIDS. J Neurovirol. 2010;16(5):355-367.

24. Churchill M, Nath A. Where does HIV hide? A focus on the central nervous system. Curr Opin HIV AIDS. 2013;8(3):165-169.

25. Joseph SB, Arrildt KT, Swanstrom AE, Schnell G, Lee B, Hoxie JA, Swanstrom R. Quantification of entry phenotypes of macrophage-tropic HIV-1 across a wide range of CD4 densities. J Virol. 2014;88(4):1858-1869.

26. Musich T, O’Connell O, Gonzalez-Perez MP, Derdeyn CA, Peters PJ, Clapham PR. HIV-1 non-macrophage-tropic R5 envelope glycoproteins are not more tropic for entry into primary CD4+ T-cells than envelopes highly adapted for macrophages. Retrovirology. 2015;12:25. 
27. Quitadamo B, Peters PJ, Repik A, O’Connell O, Mou Z, Koch M, Somasundaran M, Brody R, Luzuriaga K, Wallace A, Wang S, Lu S, McCauley S, Luban J, Duenas-Decamp M, Gonzalez-Perez MP, Clapham PR. HIV-1 R5 macrophage-tropic envelope glycoprotein trimers bind CD4 with high affinity, while the CD4 binding site on Non-macrophage-tropic, T-tropic R5 envelopes is occluded. J Virol. 2018;92(2).pii: e00841-17.

28. Bracq L, Xie M, Lambelé M, Vu LT, Matz J, Schmitt A, Delon J, Zhou P, Randriamampita C, Bouchet J, Benichou S. T cellmacrophage fusion triggers multinucleated giant cell formation for HIV-1 spreading. J Virol. 2017;91(24):e01237-17.

29. Bain LE, Nkoke C, Noubiap JJN. UNAIDS 90-90-90 targets to end the AIDS epidemic by 2020 are not realistic: comment on "Can the UNAIDS 90-90-90 target be achieved? A systematic analysis of national HIV treatment cascades”. BMJ Glob Health. 2017;2(2):e000227.

30. Maurya VK, Kumar S, Saxena SK. Molecular mechanism of neuropathogenesis and current therapeutics for neuroAIDS. Research Reports 2018;1:e1-e8.

31. Watkins CC, Treisman GJ. Cognitive impairment in patients with AIDS -prevalence and severity. HIV/AIDS - Research and Palliative Care. 2015;7:35-47.

32. Yilmaz A, Price RW, Gisslén M. Antiretroviral drug treatment of CNS HIV-1 infection. J Antimicrob Chemother. 2012;67(2):299-311.

33. Das MK, Sarma A, Chakraborty T. Nano-ART and NeuroAIDS. Drug Deliv Transl Res. 2016;6(5):452-472.

34. Zhang YL, Ouyang YB, Liu LG, Chen DX. Blood-brain barrier and neuro-AIDS. Eur Rev Med Pharmacol Sci. 2015;19(24):49274939.

35. Mahajan SD, Aalinkeel R, Law WC, Reynolds JL, Nair BB, Sykes DE, Yong KT, Roy I, Prasad PN, Schwartz SA. Anti-HIV-1 nanotherapeutics: promises and challenges for the future. Int J Nanomedicine. 2012;7:5301-5314.

36. Varghese NM, Senthil V, Saxena SK. Nanocarriers for brain specific delivery of anti-retro viral drugs: challenges and achievements. J Drug Target. 2018;26(3):195-207.

37. Kaul M, Lipton SA. Experimental and potential future therapeutic approaches for HIV-1 associated dementia targeting receptors for chemokines, glutamate and erythropoietin. Neurotox Res. 2005;8(1-2):167-186.

38. Saxena SK, Tiwari S, Nair MP. Nanotherapeutics: emerging competent technology in neuroAIDS and CNS drug delivery. Nanomedicine (Lond). 2012;7(7):941-944.

39. Nowacek A, Gendelman HE. NanoART, neuroAIDS and CNS drug delivery. Nanomedicine (Lond). 2009;4(5):557-574.

40. Nair M, Jayant RD, Kaushik A, Sagar V. Getting into the brain: Potential of nanotechnology in the management of NeuroAIDS. Adv Drug Deliv Rev. 2016;103:202-217.

41. das Neves J, Amiji MM, Bahia MF, Sarmento B. Nanotechnologybased systems for the treatment and prevention of HIV/AIDS. Adv Drug Deliv Rev. 2010;62(4-5):458-477.

42. Chhabra R, Tosi G, Grabrucker AM. Emerging use of nanotechnology in the treatment of neurological disorders. Curr Pharm Des. 2015;21(22):3111-3130.

43. Banik BL, Fattahi P, Brown JL. Polymeric nanoparticles: the future of nanomedicine. Wiley Interdiscip Rev Nanomed Nanobiotechnol. 2016;8(2):271-299.

44. Sagar V, Pilakka-Kanthikeel S, Pottathil R, Saxena SK, Nair $M$. Towards nanomedicines for neuroAIDS. Rev Med Virol. 2014;24(2):103-124.
45. Kumar L, Verma S, Prasad DN, Bhardwaj A, Vaidya B, Jain AK. Nanotechnology: a magic bullet for HIV AIDS treatment. Artif Cells Nanomed Biotechnol. 2015;43(2):71-86.

46. Ramanathan R, Jiang Y, Read B, Golan-Paz S, Woodrow KA. Biophysical characterization of small molecule antiviral-loaded nanolipogels for HIV-1 chemoprophylaxis and topical mucosal application. Acta Biomater. 2016;36:122-131.

47. Nair M, Guduru R, Liang P, Hong J, Sagar V, Khizroev S. Externally controlled on-demand release of anti-HIV drug using magneto-electric nanoparticles as carriers. Nat Commun. 2013;4:1707.

48. Saiyed ZM, Gandhi NH, Nair MP. Magnetic nanoformulation of azidothymidine 5'-triphosphate for targeted delivery across the blood-brain barrier. Int J Nanomedicine. 2010;5:157-166.

49. Yadav HKS, Al Halabi NA, Alsalloum GA. Nanogels as Novel Drug Delivery Systems - A Review. J Pharm Pharm Res. 2017;1:5.

50. Vinogradov SV, Poluektova LY, Makarov E, Gerson T, Senanayake MT. Nano-NRTIs: efficient inhibitors of HIV type-1 in macrophages with a reduced mitochondrial toxicity. Antivir Chem Chemother. 2010;21(1):1-14.

51. Batrakova EV, Gendelman HE, Kabanov AV. Cell-mediated drug delivery. Expert Opin Drug Deliv. 2011;8(4):415-433.

52. Lu W. Adsorptive-mediated brain delivery systems. Curr Pharm Biotechnol. 2012;13(12):2340-2348.

53. Rao KS, Reddy MK, Horning JL, Labhasetwar V. TAT-conjugated nanoparticles for the CNS delivery of anti-HIV drugs. Biomaterials. 2008;29(33):4429-4438.

54. Wang W, Ye C, Liu J, Zhang D, Kimata JT, Zhou P. CCR5 gene disruption via lentiviral vectors expressing Cas9 and single guided RNA renders cells resistant to HIV-1 infection. PLoS One. 2014;9(12):e115987.

55. Yi X, Manickam DS, Brynskikh A, Kabanov AV. Agile delivery of protein therapeutics to CNS. J Control Release. 2014;190:637663.

56. Schwarze SR, Hruska KA, Dowdy SF. Protein transduction: unrestricted delivery into all cells? Trends Cell Biol. 2000;10(7):290-295.

57. Hersh DS, Wadajkar AS, Roberts N, Perez JG, Connolly NP, Frenkel V, Winkles JA, Woodworth GF, Kim AJ. Evolving drug delivery strategies to overcome the blood brain barrier. Curr Pharm Des. 2016;22(9):1177-1193.

58. Kuo YC, Kuo CY. Electromagnetic interference in the permeability of saquinavir across the blood-brain barrier using nanoparticulate carriers. Int J Pharm. 2008;351(1-2):271-281.

59. Spitzenberger TJ, Heilman D, Diekmann C, Batrakova EV, Kabanov AV, Gendelman HE, Elmquist WF, Persidsky Y. Novel delivery system enhances efficacy of antiretroviral therapy in animal model for HIV-1 encephalitis. J Cereb Blood Flow Metab. 2007;27(5):1033-1042.

60. De Schutter C, Ehteshami M, Hammond ET, Amblard F, Schinazi RF. Metabolism of Nucleosides and Nucleotides Prodrugs. Curr Pharm Des. 2017;23(45):6984-7002.

61. Chan L, Asriel B, Eaton EF, Wyatt CM. Potential kidney toxicity from the antiviral drug tenofovir: new indications, new formulations, and a new prodrug.Curr Opin Nephrol Hypertens. 2018;27(2):102-112.

62. Dalpiaz A, Fogagnolo M, Ferraro L, Capuzzo A, Pavan B, Rassu G, Salis A, Giunchedi P, Gavini E. Nasal chitosan microparticles target a zidovudine prodrug to brain HIV sanctuaries. Antiviral Res. 2015;123:146-157. 